\title{
Kinematics and workspace analysis of a robotic device for performing rehabilitation therapy of upper limb in stroke-affected patients
}

\author{
TONY PUnNoOSE VALAYIL ${ }^{1 *}$, Rose ShaJI AUgustinE ${ }^{2}$ \\ ${ }^{1}$ Department of Mechanical Engineering, Coimbatore Institute of Technology, Coimbatore Tamil Nadu 641014, India. \\ ${ }^{2}$ Department of Civil Engineering, Rathinam Technical Campus, Coimbatore, Tamil Nadu 641021, India.
}

\begin{abstract}
Purpose: Exoskeleton robots generally have multi-functions and one such function is doing rehabilitation therapy in upper limb and lower limb in stroke-affected patients. A novel hybrid (serial-parallel) robot manipulator was proposed in this paper for rehabilitation of upper limb and its kinematics are studied systematically. This robot manipulator intends to perform wrist flexion, wrist extension, wrist radial deviation, wrist ulnar deviation, elbow flexion, elbow extension, elbow pronation and elbow supination motions. The contemporary mechanical designs especially the kinematic structure of upper limb exoskeleton robots have a unique feature that is, almost all of them use serial manipulators, and few others used parallel manipulators. The kinematic structure of the proposed robot is that of a hybrid manipulator (two parallel manipulators connected in series) which has 4-degrees-of-freedom. It is composed of an upper 3SPS-type parallel manipulator and 2SPR-type parallel manipulator connected in series. Methods: The Jacobian and Hessian Matrix method was used to derive the manipulator kinematic formula for solving the displacement, velocity and acceleration. Results: A 3D model of the robotic arm was constructed and analyzed by simulation. The positioning workspace of manipulator was constructed and analyzed. Conclusions: The 3SPS-type parallel manipulator has good kinematic characteristics while performing wrist motions. The 2SPR-type parallel manipulator produced singular configuration, while performing the desired rehabilitation elbow motions, it was found to not be suitable for usage in performing rehabilitation therapy in stroke-affected patients.
\end{abstract}

Key words: parallel manipulator, kinematics, exoskeleton, rehabilitation, stroke, patients

\section{Introduction}

A robotic system consists of a mechanical manipulator made up of kinematic links and joints. The mechanical manipulators can be classified based on the kinematic structure as serial manipulator, parallel manipulator, and hybrid manipulator. Serial manipulator has kinematic links connected in series. Parallel manipulator has kinematic links connected independently. Hybrid manipulator is a combination of serial manipulator and parallel manipulator. Hybrid manipulator also possesses a combination of two parallel manipulators connected in series. Due to this serial interconnection between parallel manipulators, the hybrid manipulator holds the advantages of both serial manipulator and parallel manipulator. The parallel manipulator has larger load-to-weight ratio and stiffness than serial manipulator, whereas the serial manipulator has larger workspace and more flexibility than the parallel manipulator [20].

In medical field, robots are used for performing rehabilitation therapy in stroke-affected patients. ASPIRE robot is a spherical-parallel robot used for post-stroke rehabilitation therapy for upper limb. The parallel architecture with spherical structure of ASPIRE robot enables better motion in the entire robot workspace. It performs shoulder flexion, shoulder exten-

\footnotetext{
* Corresponding author: Tony Punnoose Valayil, Coimbatore Institute of Technology, Coimbatore, 641014, India. Phone: +91-9489911023, e-mail: tonyisdesigning@gmail.com

Received: June 20th, 2021

Accepted for publication: October 15th, 2021
} 
sion, shoulder abduction, shoulder adduction, forearm pronation and forearm supination motions. Kinematic analysis and singular configuration of the ASPIRE robot were determined [21]. A planar cable-driven parallel robot called as "RehaBot" was developed for poststroke rehabilitation. This robot was inspired based on mirror therapy. Mirror therapy makes use of mirrorillusion which mimics the paretic limb movements. It perform all upper limb motions [22]. Alamdari and Krovi [23] designed a cable-driven rehabilitation robot for upper limb. It has five cables and one actuator. The kinematic analysis of the robot was solved using transformation matrix. A low-cost trajectory tracking controller was designed for tracking the trajectory of the robot. This robot is named as PACER (Parallel Articulated-Cable Exercise Robot). Dynamic analysis was done using Newton-Euler formulation. This robot performs forearm pronation/supination, flexion/extension of the wrist, abduction/adduction of the arm and flexion/extension of the arm.

In recent years, many exoskeleton robots were developed for upper limb rehabilitation and researchers have reviewed all of it, but focus was not given to the mechanical design of active exoskeleton robots performing passive therapy. Active robots are robots powered using an external source such as an electric motor. They can be used for performing passive therapy, a therapy where human effort is not needed. Hence, in Table 1, the proposed hybrid robot was compared with existing active robots which perform passive therapy in stroke-affected patients. The comparison was made based on active degrees-of-freedom (DOF), kinematic structure, power transmission method, type of actuator used, and rehabilitation applied on selected joints and supported movements. Aside from the 7-DOF in the upper limb, there are also several passive DOF (mentioned as "+1-DOF" in Table 1). Some robots also perform rehabilitation therapy for the hand. These DOF are not acceptable for therapy since they are difficult to control and can injure patients.

Literature review suggests that, for rehabilitation of upper limb in stroke-affected patients, a hybrid exoskeleton manipulator (having two parallel manipulators

Table 1. Comparison between existing active exoskeleton robots versus proposed hybrid robot

\begin{tabular}{|c|c|c|c|c|c|}
\hline Name & Active DOF & $\begin{array}{l}\text { Kinematic } \\
\text { structure }\end{array}$ & $\begin{array}{l}\text { Power } \\
\text { transmission } \\
\text { method }\end{array}$ & $\begin{array}{l}\text { Actuator } \\
\text { type }\end{array}$ & $\begin{array}{c}\text { Applied joints and } \\
\text { supported movements }\end{array}$ \\
\hline RiceWrist [4] & 4 & PM & LM, CD & EM & Ew (F-E, P-S), Wt (F-E, U-R) \\
\hline Armeo [15] & 6 & SM & LM & EM & Sr (F-E, I-E, Ab-Ad), Ew (F, S), Wt (F) \\
\hline MAHI [3] & 5 & PM & LM, CD & EM & $\begin{array}{l}\text { Ew (P-S, F-E), Wt (U-R, F-E) } \\
\text { Wt (+1-DOF) }\end{array}$ \\
\hline $\begin{array}{l}\text { Pineda-Rico } \\
\text { et al. [17] }\end{array}$ & 8 & SM & LM & EM & $\begin{array}{l}\text { Sr (F-E, I-E, Ab-Ad), Ew (P-S, F-E), } \\
\text { Wt (U-R, F-E), Sr (+1- DOF) }\end{array}$ \\
\hline ETS-MARSE [19]] & 7 & SM & LM, GD & EM & $\begin{array}{l}\text { Sr (F-E, I-E, Ab-Ad), Ew (P-S, F-E), } \\
\text { Wt (U-R, F-E) }\end{array}$ \\
\hline ARMin [13] & 6 & SM & LM, CD & EM & $\begin{array}{l}\text { Sr (I-E, Ab-Ad, F-E), Ew (P-S, F-E), } \\
\text { Wt (F-E) }\end{array}$ \\
\hline UL-Exo7 [1] & 7 & SM & LM & EM & $\begin{array}{l}\text { Sr (Ab-Ad, F-E, I-E), Ew (P-S, F-E), } \\
\text { Wt (F-E, U-R) }\end{array}$ \\
\hline Gentle/G [8] & 9 & PM & LM, CD & EM & $\begin{array}{l}\text { Sr (Ab-Ad, F-E, I-E), Wt ( F-E, U-R), } \\
\text { Ew (P-S, F-E), Thumb ( +1-DOF), } \\
\text { Fingers (+1-DOF) }\end{array}$ \\
\hline CADEN-7 [16] & 7 & SM & LM, CD, GD & EM & $\begin{array}{l}\text { Sr (Ab-Ad, F-E, I-E), Ew (P-S, F-E), } \\
\text { Wt (F-E, U-R) }\end{array}$ \\
\hline Wrist Gimbal [11] & 3 & SM & $\mathrm{CD}, \mathrm{GD}$ & EM & Ew (P-S), Wt ( F-E, U-R), \\
\hline KINARM [14] & 2 & SM & LM & EM & Sr (Ab-Ad), Ew (F-E), \\
\hline BONES [12] & 6 & PM & LM & PA & $\begin{array}{l}\text { Sr (Ab-Ad, F-E, I-E), Ew (P-S, F-E), } \\
\text { Wt (F-E), }\end{array}$ \\
\hline $\begin{array}{l}\text { Proposed } \\
\text { Hybrid Robot }\end{array}$ & 4 & $\begin{array}{c}\text { Hybrid } \\
\text { Manipulator }\end{array}$ & $L M, G D$ & $E M, P A$ & $E w(P-S, F-E), W t(F-E, U-R)$ \\
\hline
\end{tabular}

Supported movements for upper limb (F-E - flexion-extension; Ab - Ad - abduction-adduction, I-E - internal-external, P-S - pronation-supination, U-R - ulnar-radial. Applied joint ( $\mathrm{Sr}$ - shoulder, Ew - elbow, Wt - wrist). Power transmission method (LM - linkage mechanism, CD - cable drive, GD - gear drive), Actuator type (EM - electric motor, PA - pneumatic actuator, HA - hydraulic actuator). $\mathrm{PM}$ - parallel manipulator, $\mathrm{SM}$ - serial manipulator. 
connected in series) has not been invented yet. Thus, the authors were determined to design a hybrid manipulator for performing human wrist motions and elbow motions during rehabilitation therapy for upper limb in stroke-affected patients. Electric motors and pneumatic actuators are the actuator types used. Linkage mechanism and gear drive provides the required power transmission.

\section{Materials and methods}

\subsection{Structural characteristics of the hybrid manipulator}

The hybrid manipulator is composed of an upper 3SPS-type ( $\mathrm{S}$ - spherical joint, $\mathrm{P}$ - prismatic joint, $\mathrm{R}$ - revolute joint) $\mathrm{PM}$ (parallel manipulator) and a lower 2SPR-type PM which are connected in series as shown in Fig. 1. The upper PM and lower PM have a top platform and a fixed base platform. This hybrid manipulator can be fixed to the human arm at three points. The top platform of upper PM is fixed at the wrist then the top platform of lower PM is fixed at the center of the forearm and, finally, the base platform of lower PM is fixed at the center of the upper arm, which is illustrated in Figs. 2 and 3. Velcro strapping and ergonomic palm splint can be used for maintaining the desired alignment, thereby preserving natural movement of the human arm. Thus the kinematic structure of the robot manipulator aligns with that of the human arm. The proposed hybrid exoskeleton manipulator shown in Fig. 1 has dimensions in accordance with the upper limb of a 44-year old male patient.

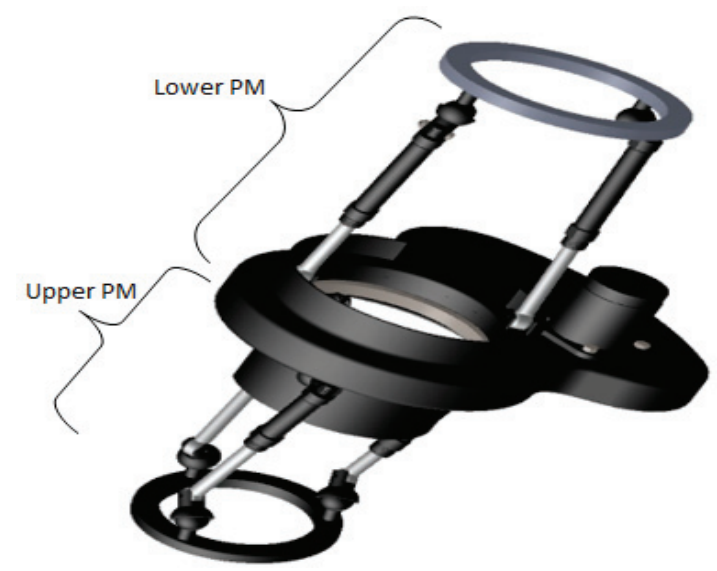

Fig. 1. 3D model of the serial-parallel robotic arm

The upper PM performs wrist flexion, wrist extension, wrist radial deviation, and wrist ulnar deviation motions, as shown in Fig. 2.

The lower PM performs elbow supination, elbow pronation, elbow flexion and elbow extension motions as shown in Fig. 3.

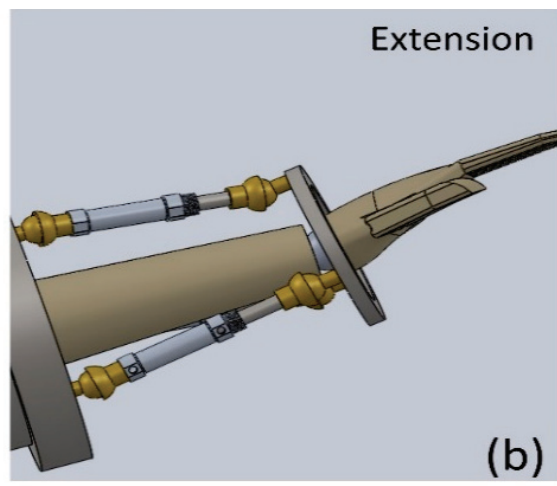

(a)
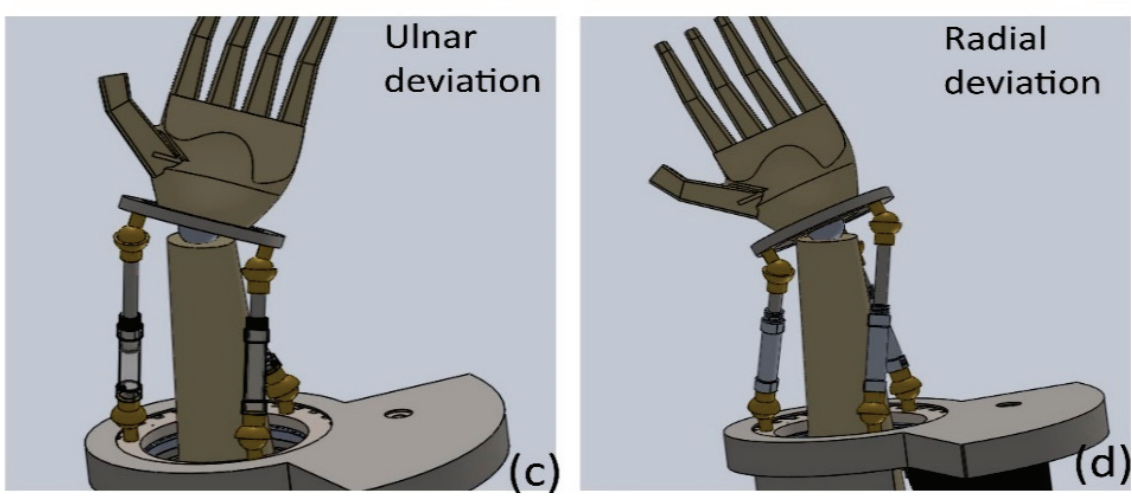

Fig. 2. Four motions performed by wrist: (a) flexion, (b) extension, (c) ulnar deviation, (d) radial deviation 

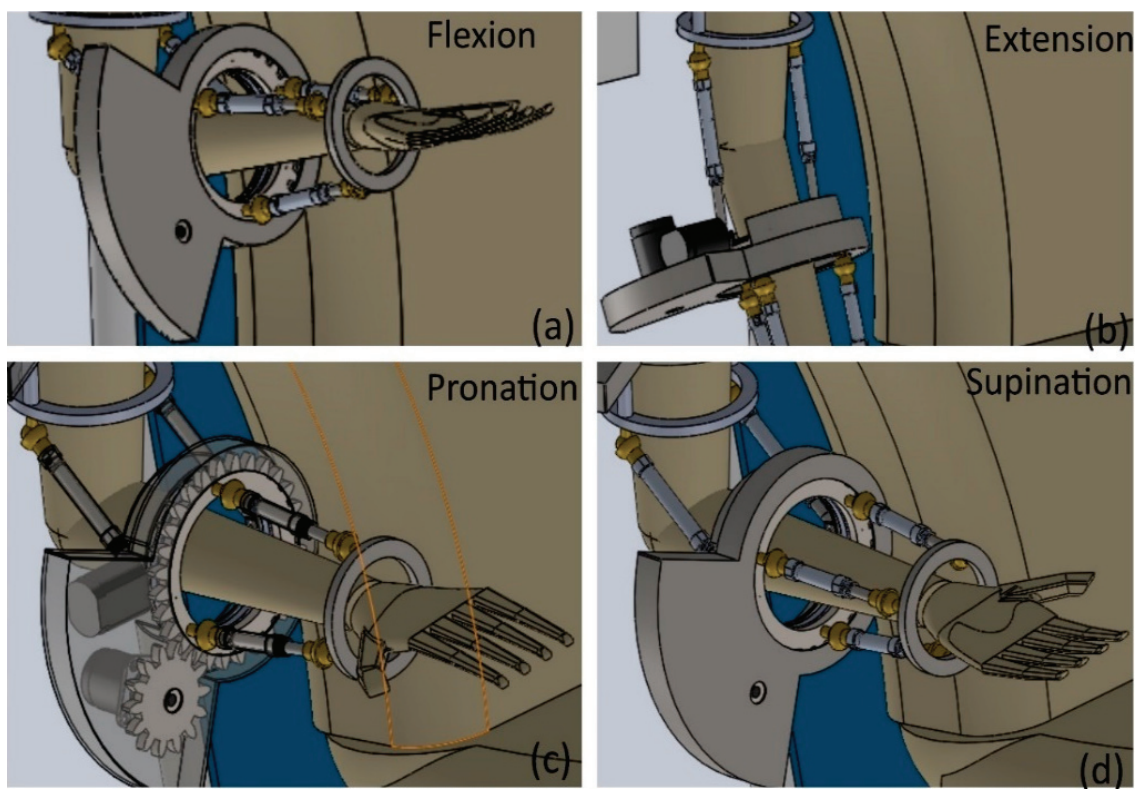

Fig. 3. Four motions performed by the elbow: (a) flexion, (b) extension, (c) pronation, (d) supination

In the kinematic model (Fig. 4 left), the upper PM has a top platform $\left(m_{2}\right)$ with center $o_{2}$ and has a base platform $\left(m_{1}\right)$ with center $o_{1}$. Both the platforms are connected through 3SPS-type linear active pneumatic legs $r_{1}, r_{2}$, and $r_{3}$. The leg $r_{1}$ connects spherical joints $b_{1}$ and $B_{1}$. Similarly, $r_{2}$ connects spherical joints $b_{2}$ and $B_{2}$ and $r_{3}$ connects spherical joints $b_{3}$ and $B_{3}$. Spherical joints are placed at equidistant from each other. Thus, the platforms $m_{2}$ and $m_{1}$ are considered as equilateral triangular shapes in kinematic model. Each platform has three sides $l_{i}(i=1,2,3)$ and $L_{i}(i=1,2,3)$ in the kinematic model.

The lower PM (Fig. 4 right), has a top platform $(m)$ with center $o$ and base platform $(B)$ with center $O$. They are connected by 2SPR-type linear active pneumatic legs $r_{11}$ and $r_{21}$. Thus, $r_{11}$ connects revolute joint $b_{11}$ and spherical joint $B_{11}$. Similarly, $r_{21}$ connects revolute joint $b_{21}$ and spherical joint $B_{21}$. In lower PM (Fig. 4 right), revolute joints (denoted by R) and spherical joints (denoted by $\mathrm{S}$ ) are placed diametrically opposite to each

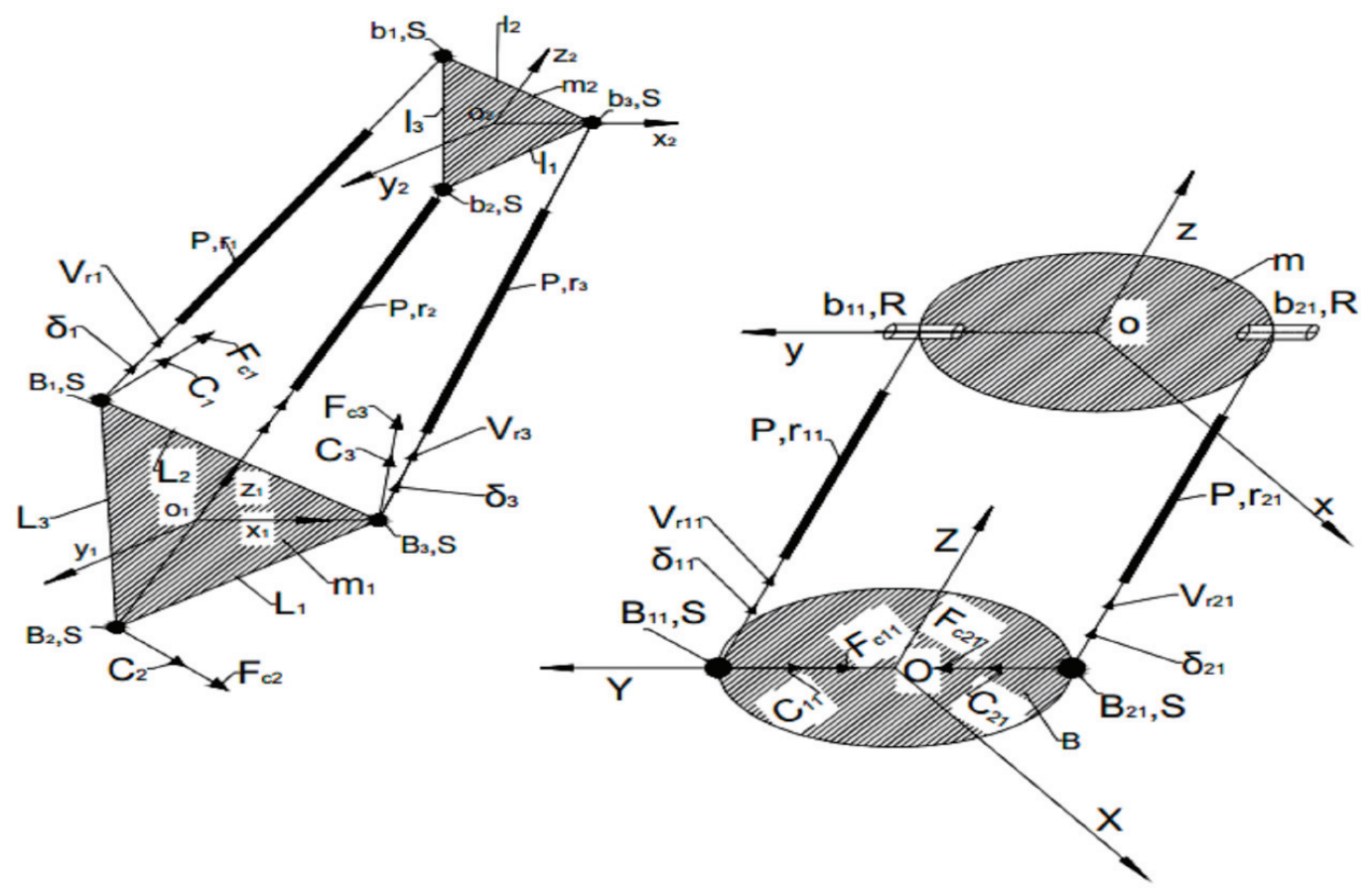

Fig. 4. Kinematic model of upper PM (left) and lower PM (right) 
Table 2. Achieved ROM versus standard ROM

\begin{tabular}{|c|c|c|c|c|c|c|c|c|}
\hline \multirow{2}{*}{$\begin{array}{c}\text { Upper Limb motions } \\
\text { Motion }\end{array}$} & \multicolumn{4}{|c|}{ Wrist motions } & \multicolumn{4}{|c|}{ Elbow motions } \\
\hline & 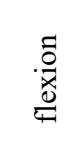 & 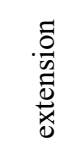 & . & 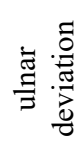 & $\begin{array}{l}\stackrel{0}{\vec{x}} \\
\stackrel{0}{e}\end{array}$ & $\begin{array}{l}\frac{\Xi}{0} \\
\frac{0}{0} \\
\frac{0}{x} \\
0 \\
0\end{array}$ & 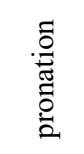 & .: \\
\hline Achieved ROM [ํ] & $0-17$ & $0-5$ & $0-8$ & $0-6$ & $0-98$ & $98-0$ & $0-80$ & $0-70$ \\
\hline Standard ROM [ [ ] [18] & $0-90$ & $0-80$ & $0-15$ & $0-40$ & $0-146$ & $146-0$ & $0-80$ & $0-85$ \\
\hline
\end{tabular}

other, so the platforms $m$ and $B$ are considered having a circular shape in kinematic model.

The prismatic actuators in upper PM performs all wrist motions and the prismatic actuators of lower PM performs elbow flexion/extension motions. The gear drive in lower PM manipulator performs elbow pronation and supination motion.

Comparison between the standard range of motion (ROM) of the human upper limb while performing wrist and elbow motions versus the achieved ROM using the proposed hybrid manipulator are given in Table 2. The achieved ROM was measured during simulation in Solidworks software package using the hybrid manipulator 3D model attached to a human arm, as shown in Figs. 2, 3. A human upper limb was modelled in Solidworks software and inserted into the manipulator.

When the manipulator performed the desired motions, the point of contact between the manipulator and the human arm was marked-down. There is supposed to be only three points of contact between upper limb and the robot manipulator. These are the fixed positions of manipulator when fixed with the human upper limb. While performing the desired motion, if any other part of the manipulator touches human upper limb, it was considered a contact point. The orientation of the manipulator platforms from mean position was measured which is the achieved ROM.

The base platform of the upper PM also acts as the top platform for lower PM. Wrist flexion motion and wrist extension motion together are 1-DOF motion performed by hybrid manipulator. Similarly, other 3-DOF performed are wrist ulnar deviation/radial deviation, elbow flexion/extension and elbow pronation/supination motions. The 2-DOF of lower PM are independently controlled by actuators. Hence, in the lower PM, since two different types of actuators (pneumatic and gear drive) are used, elbow flexion/extension and elbow pronation/supination can be done simultaneously. In case of upper PM, only 1-DOF can be performed at the same time since the same pneumatic actuators are used for performing 2-DOF. However, for instance, wrist flexion and elbow flexion can be performed at the same time.

\subsection{Displacement of upper PM}

When the upper PM performs wrist flexion/extension motions and wrist radial deviation/ulnar deviation motions, the degree of orientation of the top platform is analyzed from the 3D model through simulation. It is taken as input for computing inverse displacement analysis.

If $\left\{m_{2}\right\}: o_{2}-x_{2} y_{2} z_{2}$ and $\left\{m_{1}\right\}: o_{1}-x_{1} y_{1} z_{1}$ are local and global coordinate frames attached to the top platform $\left(m_{2}\right)$ and base platform $\left(m_{1}\right)$, " $D$ " and " $d$ " are the radii of the top platform and fixed base platform, Eq. (1) is used to compute the position of all the spherical joints $\left(b_{1}, b_{2}\right.$, and $\left.b_{3}\right)$ w.r.t the top platform $\left\{m_{2}\right\}$ [10]. Thus, the position vectors of $b_{1}, b_{2}$, and $b_{3}$ w.r.t $\left\{m_{2}\right\}$ can be obtained. Taking $D=52.50 \mathrm{~mm}$, which is the distance from $o_{2}$ to $b_{1}, o_{2}$ to $b_{2}$ and $o_{2}$ to $b_{3}$, respectively, we can obtain:

$$
\begin{gathered}
X_{b_{1}}=(-D) / 2 ; \quad Y_{b_{1}}=(\sqrt{3} D) / 2 ; \quad Z_{b_{1}}=0 ; \\
X_{b_{2}}=(-D) / 2 ; \quad Y_{b_{2}}=(-\sqrt{3} D) / 2 ; \quad Z_{b_{2}}=0 ; \\
X_{b_{3}}=D ; \quad Y_{b_{3}}=0 ; \quad Z_{b_{3}}=0 .
\end{gathered}
$$

The position vectors of spherical joints $B_{1}, B_{2}$, and $B_{3}$ w.r.t $\left\{m_{1}\right\}$ can be computed by replacing " $D$ " by " $d$ " in Eq. (1), where $d=67.5 \mathrm{~mm}$ is the distance from $o_{1}$ to $B_{1}, o_{1}$ to $B_{2}$ and $o_{1}$ to $B_{3}$. Next, the position vectors of $b_{1}, b_{2}$ and $b_{3}$ w.r.t $\left\{m_{1}\right\}$ can be obtained using Eq. (2) [9].

$$
{ }^{m_{1}} b_{i}={ }^{m_{1}} R_{m_{2}}{ }^{m_{2}} b_{i}+{ }^{m_{1}} o_{2} \quad(i=1,2,3),
$$

where, ${ }^{m_{1}} R_{m_{2}}$ is the transformation matrix from $\left\{m_{2}\right\}$ to $\left\{m_{1}\right\} .{ }^{m_{1}} O_{2}$ be the position of $o_{2}$ w.r.t coordinate frame $\left\{m_{1}\right\}$.

Taking the wrist extension motion performed by upper PM for inverse displacement analysis into consideration, it can be seen that the local coordinate 
frame $\left(o_{2}-x_{2} y_{2} z_{2}\right)$ is rotated by $6.68^{0}$ about $y_{2}$-axis in clockwise direction to align with the global coordinate frame $\left(o_{1}-x_{1} y_{1} z_{1}\right)$. This is determined through simulation when the upper PM performs maximum wrist extension motion of 5 degrees, which is the achieved ROM given in Table 2. The corresponding rotation angle and the axis of rotation are submitted in rotation matrix and hence obtained ${ }^{m_{1}} R_{m_{2}}$ as follows:

$$
\begin{gathered}
{ }_{m_{1}} R_{m_{2}}\left(y_{2}, 6.68^{0}\right) \\
=[\cos (6.68), 0, \sin (6.68) ; 0,1,0 ; \\
-\sin (6.68), 0, \cos (6.68)] .
\end{gathered}
$$

The top platform moves while performing wrist motions, whereas the bottom platform is fixed. The position of $o_{2}$ w.r.t $o_{1}$ does not change for any of the wrist motions because there is only angular movement and no translational movement of the top platform. Hence, the position of ${ }^{m_{1}} o_{2}$ remains constant in all axis and given as follows:

$$
{ }^{m_{1}} o_{2}=\left[x_{0} ; y_{0} ; z_{0}\right]=[0 ; 0 ; 184.21] \mathrm{mm} .
$$

Then, substituting ${ }^{m_{2}} b_{1},{ }^{m_{2}} b_{2},{ }^{m_{2}} b_{3}$ in Eq. (2), ${ }^{m_{1}} b_{1}$, ${ }^{m_{1}} b_{2}$ and ${ }^{m_{1}} b_{3}$ can be obtained. The length of the legs of 3SPS-type PM in $\left\{m_{1}\right\}$ is represented in Eq. (3) [9]

$$
{ }^{m_{1}} r_{i}={ }^{m_{1}} b_{i}-{ }^{m_{1}} B_{i} \quad(i=1,2,3) .
$$

In Equation (3), " $r_{i}$ " represents the length of the active pneumatic legs of upper PM. The length of each leg in $\left\{m_{1}\right\}$ is ${ }^{m_{1}} r_{1},{ }^{m_{1}} r_{2}$, and ${ }^{m_{1}} r_{3}$, which can be obtained by submitting the results obtained for position vectors of $B_{1}, B_{2}$, and $B_{3}$ w.r.t $\left\{m_{1}\right\}$ from Eq. (1) and also position vectors of $b_{1}, b_{2}$, and $b_{3}$ w.r.t $\left\{m_{1}\right\}$ from Eq. (2) in Eq. (3). Thus, inverse displacement analysis of upper PM is fully solved for wrist extension motion.

Equations (2), (3) are also used to solve the displacements of the legs during wrist flexion, wrist radial deviation and wrist ulnar deviation motions. The change that occurs in all cases is the rotation matrix ${ }^{m_{1}} R_{m_{2}}$. It is observed through simulation that the local frame $\left(o_{2}-x_{2} y_{2} z_{2}\right)$ is rotated about $y_{2}$-axis by $-9.65^{0}$ to align with the global frame $\left(o_{1}-x_{1} y_{1} z_{1}\right)$ for wrist flexion motion. In wrist radial deviation and wrist ulnar deviation motions, the local frame $\left(o_{2}-x_{2} y_{2} z_{2}\right)$ is rotated about $x_{2}$-axis to align with the global frame $\left(o_{1}-x_{1} y_{1} z_{1}\right)$. The rotating angles for wrist radial de- viation and wrist ulnar deviation are found to be $-5^{0}$ and $30^{\circ}$. The results for inverse displacement are shown in Section 3.

\subsection{Velocity of upper PM}

In velocity analysis, two motions performed by the upper PM are considered together. That is, wrist radial deviation and wrist ulnar deviation motions are combined and wrist flexion and wrist extension motions are combined. Thus, the rotation matrix ${ }^{m_{1}} R_{m_{2}}$ vary as the rotation angle varies.

When ${ }^{m_{1}} e_{i}$ is the vector from $o_{2}$ to $b_{i}$ in $\left\{m_{1}\right\}$, it is given as ${ }^{m_{1}} e_{i}={ }^{m_{1}} b_{i}-{ }^{m_{1}} O_{2}$. When ${ }^{m_{1}} \delta_{i}$ represents the unit vector of $r_{i}$ in $\left\{m_{1}\right\}$, it is given as ${ }^{m_{1}} \delta_{i}=\frac{{ }^{m_{1}} b_{i}-{ }^{m_{1}} B_{i}}{r_{i}}$. When ${ }^{m_{1}} c_{i}$ represents the unit vector of $f_{c i}$, where $f_{c i}$ denotes the constraint force exerted on $r_{i}$ at $b_{i},{ }^{m_{1}} c_{1}$ is given as ${ }^{m_{1}} c_{1}=\frac{{ }^{m_{1}} e_{2}-{ }^{m_{1}} e_{3}}{l_{1}}$. When ${ }^{m_{1}} V$ be the general velocity of $o_{2}$ in $\left\{m_{1}\right\}$, and $V_{r}$ is the general input velocities of the legs of upper PM, then $V_{r}$ is given in Eq. (4) as follows [9]:

$$
V_{r}=J^{m_{1}} V
$$

$V_{r}$ is a $6 \times 1$ matrix given by $V_{r}=\left[V_{r_{1}} ; V_{r_{2}} ; V_{r_{3}} ; 0\right.$; $0 ; 0]_{6 \times 1}$ and $V_{r_{1}}, V_{r_{2}}$ and $V_{r_{3}}$ are the velocities of active prismatic legs. Then, ${ }^{m_{1}} V$ is given as ${ }^{m_{1}} V=\left(\begin{array}{l}v \\ \omega\end{array}\right)^{m_{1}}$, where ${ }^{m_{1}} v$ and ${ }^{m_{1}} \omega$ represent the translational and angular velocities of $m_{2}$ near $o_{2}$ in $\left\{m_{1}\right\}$. The top platform of upper PM exhibits only angular velocity and has no translational velocity while performing the desired motions. " $J$ " is a $6 \times 6$ Jacobian matrix given as Eq. (5)

$$
J=\left[\begin{array}{cc}
\delta_{1}^{T} & \left(\delta_{1} \times e_{1}\right)^{T} \\
\delta_{2}^{T} & \left(\delta_{2} \times e_{2}\right)^{T} \\
\delta_{3}^{T} & \left(\delta_{3} \times e_{3}\right)^{T} \\
c_{1}^{T} & \left(e_{1} \times c_{1}\right)^{T} \\
c_{2}^{T} & \left(e_{2} \times c_{2}\right)^{T} \\
c_{3}^{T} & \left(e_{3} \times c_{3}\right)^{T}
\end{array}\right]^{T}
$$

The $6 \times 6$ Jacobian matrix obtained for upper PM during wrist extension motion to wrist flexion motion at time $(t=1 \mathrm{sec})$ is 


$$
J=\left[\begin{array}{cccccc}
0.0426 & -0.0720 & 0.9965 & -44.9994 & -25.7763 & 0.0643 \\
0.0426 & 0.0720 & 0.9965 & 44.9994 & -25.7763 & -0.0643 \\
-0.0806 & 0 & 0.9967 & 0 & 52.4269 & 0 \\
-0.8586 & -0.4995 & -0.1153 & -6.9815 & 0 & 51.9845 \\
0.8586 & -0.4995 & 0.1153 & -6.9815 & 0 & 51.9845 \\
0 & 1.0000 & 0 & -6.9879 & 0 & 52.0329
\end{array}\right] .
$$

During inverse velocity analysis, the velocity of the top platform is considered as input. Eq. (4) can be rewritten as ${ }^{m_{1}} V=J^{1} V_{r}$ for computing inverse velocity analysis. Substituting the values of $\delta_{i}, c_{i}$, and $e_{i}$ in Jacobian matrix, $J^{-1}$ can be obtained. During computation of forward velocity analysis, the velocities of the active legs of the upper PM is considered as input, $V_{r_{1}}=V_{r_{2}}=V_{r_{3}}=10 t \mathrm{~mm} / \mathrm{sec}$, where " $t$ " represents time. The input velocity could be increased or decreased based on the person's recovery rate during rehabilitation or as suggested by the physician. The results for forward and inverse velocity analysis are given in Section 3.

\subsection{Acceleration of upper PM}

Inverse acceleration analysis of upper PM can be computed using Eq. (6) as follows [9]:

$$
A_{r}=J^{m_{1}} A+{ }^{m_{1}} V^{T} H^{m_{1}} V \text {, }
$$

where, ${ }^{m_{1}} A$ is general acceleration of $m_{2}$ at $o_{2}$ in $\left\{m_{1}\right\}$, $A_{r}$ is general acceleration of active legs of upper PM. Then, ${ }^{m_{1}} A$ is given as, ${ }^{m_{1}} A=\left(\begin{array}{l}a \\ \varepsilon\end{array}\right)^{m_{1}}$, where ${ }^{m_{1}} a$ is translational acceleration of $m_{2}$ at $o_{2}$ in $\left\{m_{1}\right\}$ and ${ }^{m_{1} \varepsilon}$ is angular acceleration of $m_{2}$ at $o_{2}$ in $\left\{m_{1}\right\}$.

Let, $A_{r}=\left[a_{r_{1}} ; a_{r_{2}} ; a_{r_{3}} ; 0 ; 0 ; 0\right]_{6 \times 1}$ and " $H$ " denotes Hessian matrix given by Eq. (7)

$$
H=\left[h_{1} ; h_{2} ; h_{3} ; h_{c_{1}} ; h_{c_{2}} ; h_{c_{3}}\right]_{6 \times 1},
$$

where, $h_{i}=\frac{1}{r_{i}}\left[\begin{array}{cc}-\hat{\delta}_{i}^{2} & -\hat{\delta}_{i}^{2} \cdot \hat{e}_{i} \\ -\hat{e}_{i} \cdot \hat{\delta}_{i}^{2} & \hat{r}_{i} \cdot \hat{e}_{i} \cdot \hat{\delta}_{i}+\hat{e}_{i} \cdot \hat{\delta}_{i}^{2} \cdot \hat{e}_{i}\end{array}\right]$ and $h_{c i}=$ $\left[\begin{array}{cc}0 & 0 \\ 0 & \hat{e}_{i} \cdot \hat{c}_{i}\end{array}\right]$.

Skew-symmetric matrices of vectors $\delta_{i}, e_{i}$, and $c_{i}$ are given in Eq. (8):

$$
\delta_{i}=\left[\begin{array}{ccc}
0 & -\delta z_{i} & \delta y_{i} \\
\delta z_{i} & 0 & -\delta x_{i} \\
-\delta y_{i} & \delta x_{i} & 0
\end{array}\right]
$$

$$
\begin{aligned}
e_{i} & =\left[\begin{array}{ccc}
0 & -e z_{i} & e y_{i} \\
e z_{i} & 0 & -e x_{i} \\
-e y_{i} & e x_{i} & 0
\end{array}\right] \\
c_{i} & =\left[\begin{array}{ccc}
0 & -c z_{i} & c y_{i} \\
c z_{i} & 0 & -c x_{i} \\
-c y_{i} & c x_{i} & 0
\end{array}\right] .
\end{aligned}
$$

Values of $\delta_{i}, e_{i}$, and $c_{i}$ can be obtained from ${ }^{m_{1}} e_{i}$, ${ }^{m_{1}} \delta_{i}$, and ${ }^{m_{1}} c_{i}$. The values for ${ }^{m_{1}} V^{T}$ and ${ }^{m_{1}} V$ are extracted from the forward velocity analysis and then need to be submitted in Eq. (6) to find $A_{r}$. The inverse acceleration of upper PM during wrist motions for the first 6 seconds is obtained, and the corresponding results are given in Section 3.

\subsection{Displacement, velocity and acceleration analysis of lower PM}

Let $D 1=67.5 \mathrm{~mm}$ is the radius of top platform measured from $o$ to $b_{11}$ and $o$ to $b_{21}$. The position of revolute joints w.r.t top platform can be found out using Eq. (9) as follows:

$$
{ }^{m} b_{11}=[0 ;-D 1 ; 0] \mathrm{mm} \text { and }{ }^{m} b_{21}=[0 ; D 1 ; 0] \mathrm{mm} \text {. }
$$

Likewise, the positions of spherical joints w.r.t bottom platform $(B)$ in $\{B\}$ can be found out using the same Eq. (9) by replacing $D 1$ with $d 1=67.5 \mathrm{~mm}$ (radius of bottom platform).

Initially, for computing the inverse displacement analysis, the elbow flexion motion and elbow extension motion performed by the lower PM are considered and simulation is performed in Solidworks software. During elbow flexion motion, the local coordinate frame $(o-x y z)$ is rotated about $y$-axis by $82.47^{\circ}$ to align with the global coordinate frame $(O-$ $X Y Z)$ in the clockwise direction. The rotation angle is substituted in rotation matrix ${ }^{B} R_{m}$. The position of $o$ with respect to $B$ during elbow flexion motion is given as ${ }^{B} O=[146.05 ; 0 ; 117] \mathrm{mm}$. For elbow extension motion, local frame is rotated by $166.43^{\circ}$ about $y$-axis 
in the clockwise direction to align with the global coordinate frame and thus ${ }^{B} R_{m}$ can be calculated. The position of $o$ with respect to $B$ is given as ${ }^{B} O=[33.89$; $0 ; 253.37] \mathrm{mm}$.

In the case of elbow pronation motion, the local coordinate frame $o-x y z$ is rotated about $y$-axis by $90^{\circ}$ in the anticlockwise direction to be aligned with global frame $O-X Y Z$, and in the case of elbow supination motion, $y$-axis of local frame is rotated by about $90^{\circ}$ in the clockwise direction. Let ${ }^{B} O=[146.05 ; 0$; 117] $\mathrm{mm}$ be considered for both cases. The elbow pronation and supination motions are performed after performing elbow flexion motion, hence the distance between the local coordinate frame and global coordinate frame in $z$-axis is the same as in the case of elbow flexion motion.

In the case of a lower PM, the forward velocity analysis cannot be performed because the inverse of Jacobian matrix does not exist, since the determinant value of the Jacobian matrix is zero by equation ${ }^{B} V_{1}=$ $J^{-1} V_{r_{i 1}}$. It means the manipulator has singular configuration during its motion and its degree-of-freedom changes. Velocity of the active legs are assumed as $V_{r_{i 1}}=$ $[10 * t ; 10 * t ; 0 ; 0 ; 0 ; 0]$. There is only linear displacement for top platform and no angular displacement for top platform during elbow flexion and extension motions. The length of legs changes in a smooth manner during motion. During elbow pronation and supination motion there is only angular displacement for top platform. The length remains constant in this case.

\subsection{Workspace of upper PM and lower PM}

The algorithm for finding the boundaries of the workspace is based on geometrical properties of workspace. This is known as positioning workspace. In Sections 2.1 and 2.5, the positions of the joints were obtained for upper PM and lower PM and also the lengths of the active legs were obtained. These are inputs for finding the positioning workspace.

First, consider the case of wrist flexion and wrist extension motions for workspace analysis. Eq. (3) can be rewritten in the scalar form as Eq. (10) [2]:

$$
r_{i}^{2}=\left(x_{o}-u_{i}\right)^{2}+\left(y_{0}-v_{i}\right)^{2}+\left(z_{0}-w_{i}\right)^{2} ; \quad(i=1,2,3)
$$

where:

$$
\begin{aligned}
& u_{i}=x_{a i}-q_{11} x_{b i}-q_{12} y_{b i}-q_{13} z_{b i} \quad(i=1,2,3), \\
& v_{i}=y_{a i}-q_{21} x_{b i}-q_{22} y_{b i}-q_{23} z_{b i} \quad(i=1,2,3), \\
& w_{i}=z_{a i}-q_{31} x_{b i}-q_{32} y_{b i}-q_{33} z_{b i} \quad(i=1,2,3) .
\end{aligned}
$$

Inputs like $x_{o}, y_{0}, z_{0}$ can be extracted from ${ }^{m_{1}} O_{2}$. Then, $x_{a i}, y_{a i}, z_{a i}$ can be extracted from ${ }^{m_{1}} B_{1},{ }^{m_{1}} B_{2},{ }^{m_{1}} B_{3}$. Then, $q_{11}, q_{12}, q_{13}, q_{21}, q_{22}, q_{23}, q_{31}, q_{32}, q_{33}$ can be extracted from ${ }^{m_{1}} R_{m 2}$. Then $x_{b i}, y_{b i}, z_{b i}$ can be extracted from ${ }^{m_{2}} b_{1},{ }^{m_{2}} b_{2},{ }^{m_{2}} b_{3}$. Thus the maximum and minimum lengths of the active legs can be found out using Eq. (11), i.e., the maximum and minimum limits of all three legs of the upper PM are obtained. Hence, to find out the limits, the wrist flexion and extension motions are taken together. Hence, Eq. (10) can be written as Eq. (11) as follows [2]:

$$
\begin{gathered}
r_{i \max }^{2}=\left(x_{o}-u_{i}\right)^{2}+\left(y_{0}-v_{i}\right)^{2}+\left(z_{0}-w_{i}\right)^{2} \\
(i=1,2,3), \\
r_{i \text { min }}^{2}=\left(x_{o}-u_{i}\right)^{2}+\left(y_{0}-v_{i}\right)^{2}+\left(z_{0}-w_{i}\right)^{2} \\
(i=1,2,3) .
\end{gathered}
$$

Equation (11) represents a formula for concentric spheres with the center of spheres given by $C_{i}\left(u_{i}, v_{i}, w_{i}\right)$ and radius as $r_{i \max }, r_{i \min }$. Since there are three legs for the upper PM, this leads to 3 pairs of concentric spheres. The intersection of all 6 spheres at the same time, gives the positioning workspace. Thus positioning workspace of center point of the moving platform can be obtained. The next step is to take the section of this workspace. For this, the intersection of the sphere with a plane is taken, hence, 3 pairs of concentric circles can be obtained. Therefore, to obtain a section of the workspace on a $x y$ plane, Eq. (11) can be rewritten as Eq. (12):

$$
\begin{gathered}
R_{i \max }^{2}=\left(x_{o}-u_{i}\right)^{2}+\left(y_{0}-v_{i}\right)^{2}, \\
R_{i \text { min }}^{2}=\left(x_{o}-u_{i}\right)^{2}+\left(y_{0}-v_{i}\right)^{2},
\end{gathered}
$$

where, $R_{i \max }$ and $R_{i \min }$ represent the radii of concentric circles. Similarly, the workspace analysis can be done for lower PM as well. The results are plotted and given in Section 3.

\section{Results}

In Matlab, the program is compiled using the derived analytical expressions and parameters given in Table 3. The kinematic characteristics of the hybrid manipulator found and are presented in this section. The displacement, velocity and acceleration of upper PM and lower PM are solved. The positioning workspace of the center point in the top platform for upper $\mathrm{PM}$ and lower PM are also solved. 
Table 3. Given geometric parameters, input velocity and input acceleration of PM's

\begin{tabular}{|l|c|c|c|}
\hline Symbol & $\begin{array}{c}\text { Value } \\
\text { [unit] }\end{array}$ & Symbol & $\begin{array}{c}\text { Value } \\
\text { [unit] }\end{array}$ \\
\hline$D$ & $52.50[\mathrm{~mm}]$ & $V_{r_{1}}, V_{r_{2}}, V_{r_{3}}$ & $10 t, 10 t, 10 t[\mathrm{~mm} / \mathrm{sec}]$ \\
\hline$d$ & $67.5[\mathrm{~mm}]$ & $V_{r 11}, V_{r 21}$ & $10 t, 10 t[\mathrm{~mm} / \mathrm{sec}]$ \\
\hline$D 1$ & $67.5[\mathrm{~mm}]$ & ${ }^{m}{ }_{1} \varepsilon$ & $10 t\left[\mathrm{~mm} / \mathrm{sec}^{2}\right]$ \\
\hline$d 1$ & $67.5[\mathrm{~mm}]$ & ${ }^{m}{ }_{1} \omega$ & $4 t,[\mathrm{deg} / \mathrm{sec}]$ \\
\hline
\end{tabular}

The results of inverse displacement analysis for upper PM in Table 4 confirm that, when the robot performs wrist flexion to wrist extension motions, the length of legs $r_{1}$ and $r_{2}$ increases simultaneously at the same time and length of leg $r_{3}$ decreases.

Table 4. Variation of leg lengths of upper PM for varying displacement of top platform $(\theta)$ during wrist flexion motion to wrist extension motion

\begin{tabular}{|c|c|c|c|c|}
\hline $\begin{array}{c}\theta \\
\text { [degree] }\end{array}$ & $\begin{array}{c}\text { Time } \\
{[\mathrm{sec}]}\end{array}$ & $\begin{array}{c}\text { Displacement } \\
\text { of leg } r_{1} \\
{[\mathrm{~mm}]}\end{array}$ & $\begin{array}{c}\text { Displacement } \\
\text { of leg } r_{2} \\
{[\mathrm{~mm}]}\end{array}$ & $\begin{array}{c}\text { Displacement } \\
\text { of leg } r_{3} \\
{[\mathrm{~mm}]}\end{array}$ \\
\hline-9.65 & 1 & 180.46 & 180.46 & 193.62 \\
\hline-6.76 & 2 & 181.74 & 181.74 & 191.03 \\
\hline-3.89 & 3 & 183.04 & 183.04 & 188.37 \\
\hline-1.03 & 4 & 184.34 & 184.34 & 185.75 \\
\hline 1.83 & 5 & 185.65 & 185.65 & 183.14 \\
\hline 4.70 & 6 & 186.97 & 186.97 & 180.53 \\
\hline 6.68 & 7 & 187.87 & 187.87 & 178.76 \\
\hline
\end{tabular}

Results collected in Table 5 indicate that, during wrist extension to wrist flexion motions, the length of legs $r_{1}$ and $r_{2}$ decreases simultaneously at the same time and length of leg $r_{3}$ increases. It is the reverse of results in Table 4.

Table 5. Variation of leg lengths for varying displacement of top platform $(\theta)$ during wrist extension motion to wrist flexion motion

\begin{tabular}{|c|c|c|c|c|}
\hline $\begin{array}{c}\theta \\
\text { [degree] }\end{array}$ & $\begin{array}{c}\text { Time } \\
{[\mathrm{sec}]}\end{array}$ & $\begin{array}{c}\text { Displacement } \\
\text { of leg } r_{1} \\
{[\mathrm{~mm}]}\end{array}$ & $\begin{array}{c}\text { Displacement } \\
\text { of leg } r_{2} \\
{[\mathrm{~mm}]}\end{array}$ & $\begin{array}{c}\text { Displacement } \\
\text { of leg } r_{3} \\
{[\mathrm{~mm}]}\end{array}$ \\
\hline 6.68 & 1 & 187.87 & 187.87 & 178.76 \\
\hline 3.81 & 2 & 186.56 & 186.56 & 181.35 \\
\hline 0.94 & 3 & 185.25 & 185.25 & 183.95 \\
\hline-1.92 & 4 & 183.94 & 183.94 & 186.56 \\
\hline-4.78 & 5 & 182.64 & 182.64 & 189.18 \\
\hline-7.65 & 6 & 181.34 & 181.34 & 191.81 \\
\hline-9.65 & 7 & 180.46 & 180.46 & 193.62 \\
\hline
\end{tabular}

Results collected in Table 6 indicate that, when the robot performs wrist radial deviation to wrist ulnar deviation motions, the length of legs $r_{1}$ will increase and length of leg $r_{2}$ will decrease, while length of leg $r_{3}$ remains constant.

Table 6. Variation of leg lengths for varying displacement of top platform $(\theta)$ during wrist radial deviation motion to wrist ulnar deviation motion

\begin{tabular}{|c|c|c|c|c|}
\hline $\begin{array}{c}\theta \\
{[\text { degree] }}\end{array}$ & $\begin{array}{c}\text { Time } \\
{[\mathrm{sec}]}\end{array}$ & $\begin{array}{c}\text { Displacement } \\
\text { of leg } r_{1} \\
{[\mathrm{~mm}]}\end{array}$ & $\begin{array}{c}\text { Displacement } \\
\text { of leg } r_{2} \\
{[\mathrm{~mm}]}\end{array}$ & $\begin{array}{c}\text { Displacement } \\
\text { of leg } r_{3} \\
{[\mathrm{~mm}]}\end{array}$ \\
\hline-5 & 1 & 180.89 & 188.76 & 184.81 \\
\hline 0.74 & 2 & 185.41 & 184.22 & 184.81 \\
\hline 6.47 & 3 & 189.94 & 179.73 & 184.81 \\
\hline 12.21 & 4 & 194.45 & 175.33 & 184.81 \\
\hline 17.94 & 5 & 198.91 & 171.06 & 184.81 \\
\hline 23.67 & 6 & 203.26 & 167.00 & 184.81 \\
\hline 30 & 7 & 207.78 & 162.93 & 184.81 \\
\hline
\end{tabular}

Results collected in Table 7 indicate that, when the robot performs wrist ulnar deviation motion to wrist radial deviation motion, the length of leg $r_{1}$ decreases and length of leg $r_{2}$ increases, while the length of leg $r_{3}$ remains constant all the time. Thus, from inverse displacement analysis, it can be understood that, when there is a smooth variation in the displacement of the top platform while performing rehabilitation, it can be seen that there is a smooth variation in the legs. That is when displacement of $m_{2}$ vary smoothly, the displacement of $r_{i}(i=1,2,3)$ varies smoothly. It means that there is no singularity happening while performing the desired wrist motions. Thus, the 3SPS-type upper PM has good kinematic characteristics (displacement) for all wrist motions.

Table 7. Variation of leg lengths for varying displacement of top platform $(\theta)$ during wrist ulnar deviation motion to wrist radial deviation motion

\begin{tabular}{|c|c|c|c|c|}
\hline $\begin{array}{c}\theta \\
\text { [degree] }]\end{array}$ & $\begin{array}{c}\text { Time } \\
{[\mathrm{sec}]}\end{array}$ & $\begin{array}{c}\text { Displacement } \\
\text { of leg } r_{1} \\
{[\mathrm{~mm}]}\end{array}$ & $\begin{array}{c}\text { Displacement } \\
\text { of leg } r_{2} \\
{[\mathrm{~mm}]}\end{array}$ & $\begin{array}{c}\text { Displacement } \\
\text { of leg } r_{3} \\
{[\mathrm{~mm}]}\end{array}$ \\
\hline 30 & 1 & 207.78 & 162.93 & 184.81 \\
\hline 24.07 & 2 & 199.22 & 170.77 & 184.81 \\
\hline 18.34 & 3 & 194.76 & 175.03 & 184.81 \\
\hline 12.61 & 4 & 190.25 & 179.42 & 184.81 \\
\hline 5.73 & 5 & 185.72 & 184.37 & 184.81 \\
\hline 0 & 6 & 181.21 & 188.44 & 184.81 \\
\hline-5 & 7 & 180.89 & 188.76 & 184.81 \\
\hline
\end{tabular}

During forward velocity analysis of upper PM, the velocities of all three legs are varied smoothly. From Figure 5, it can be seen that the velocity of the top platform $m_{2}$ is varying smoothly, thus, the upper PM has good kinematic characteristics (velocity). 
During inverse velocity analysis, when the velocity of the top platform varies smoothly, the velocity of the legs $r_{i}(i=1,2,3)$ varies smoothly and the results are shown in Fig. 6. Normalized values of all the legs are considered to plot the results in Fig. 6.

The difference in the maximum values of velocity during forward velocity and inverse velocity, shown in Figs. 5 and 6, is due to the difference in the selected input value of velocity given in Table 3 for ${ }_{1}^{m} \varepsilon$ and ${ }_{1}^{m} \omega$. In Figure 7, when the acceleration of the top platform $m_{2}$ varies smoothly, the acceleration of the legs $r_{i}(i=1,2,3)$ varies smoothly. Thus the 3SPS-type upper PM has good kinematic characteristics (acceleration). For all the analyse, the results are plotted for 6 seconds. Normalized values of all the legs are considered to plot the results in Fig. 7. Thus, it can be

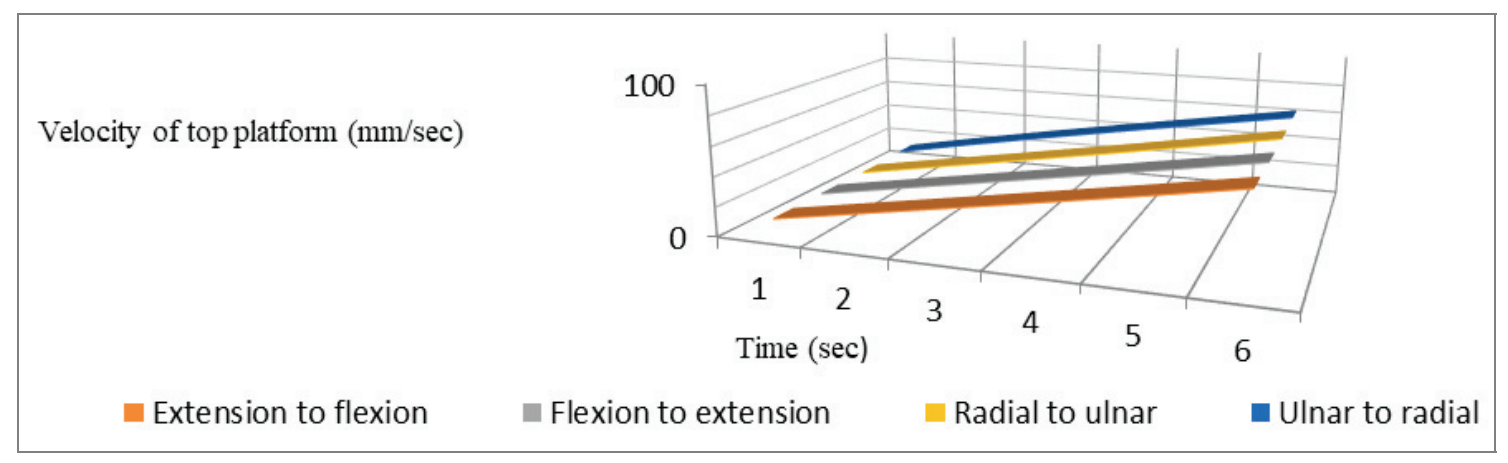

Fig. 5. Forward velocity (top platform) versus time for upper PM during wrist motions

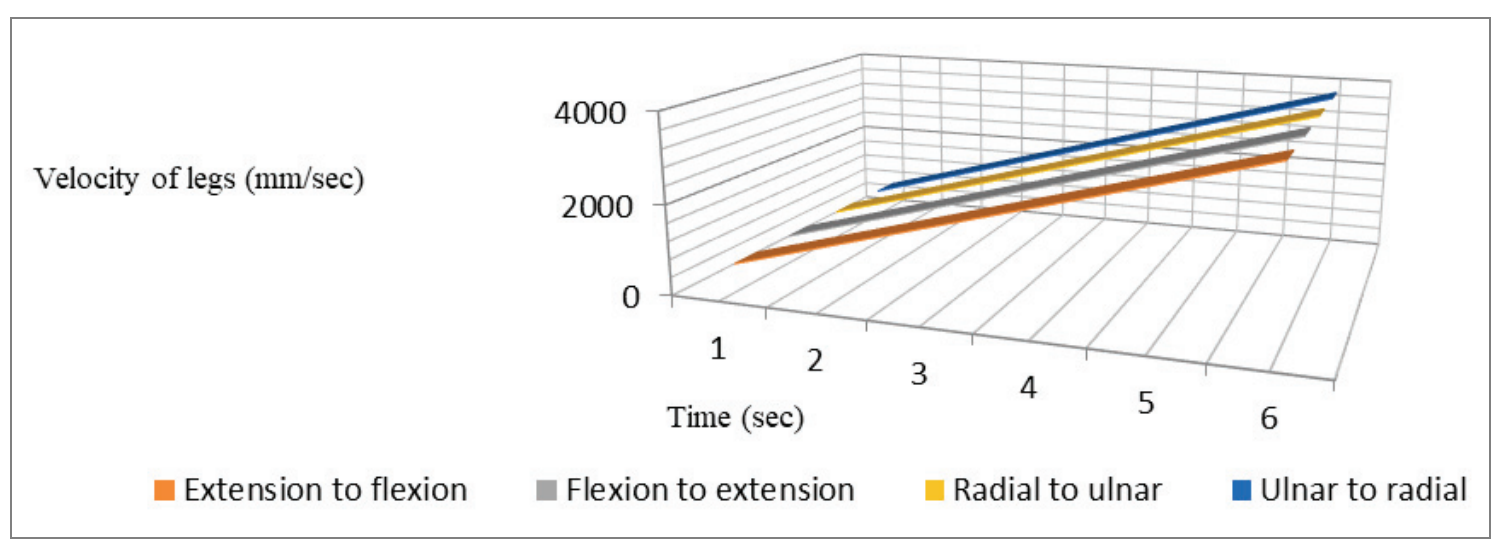

Fig. 6. Inverse velocity (legs) versus time for upper PM during wrist motions

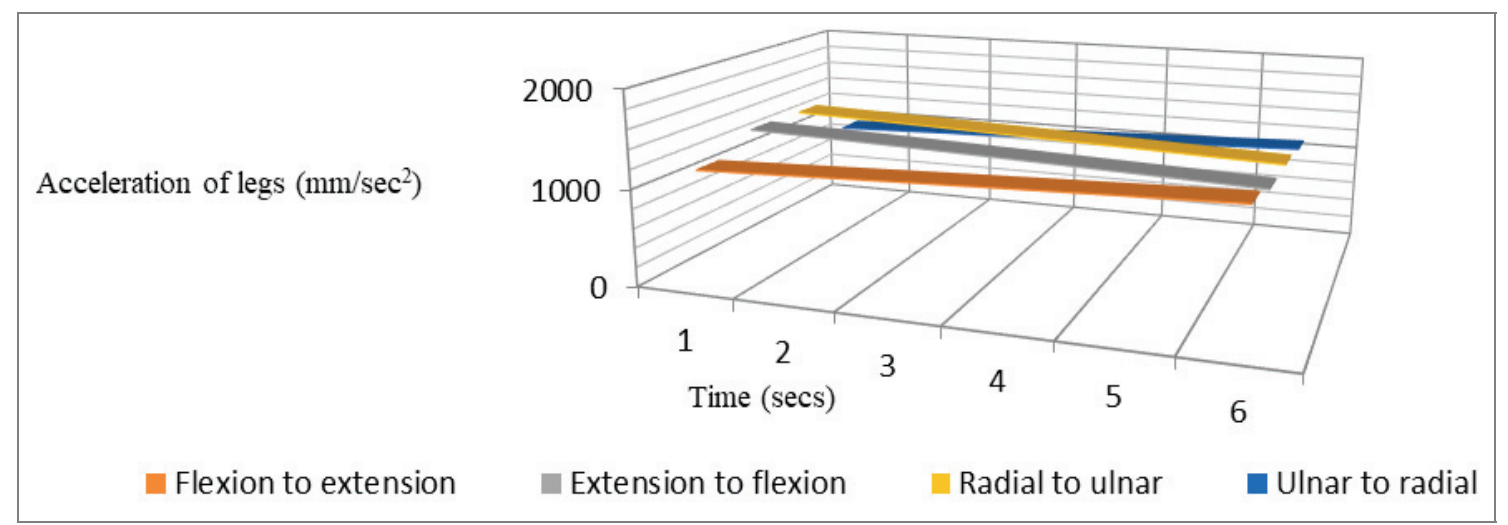

Fig. 7. Inverse acceleration (legs) versus time for upper PM 
concluded that, the 3SPS-type upper PM can be used for rehabilitation of performing wrist motions.

In Table 8, for 2SPR-type lower PM, both active legs move with same length for elbow flexion motion during rehabilitation.

Table 8. Length of legs of lower PM during inverse displacement analysis of elbow flexion motion

\begin{tabular}{|c|c|}
\hline $\begin{array}{c}\text { Length of leg } r_{11} \\
{[\mathrm{~mm}]}\end{array}$ & $\begin{array}{c}\text { Length of leg } r_{21} \\
{[\mathrm{~mm}]}\end{array}$ \\
\hline 187.135 & 187.135 \\
\hline
\end{tabular}

In Table 9, the length of the legs of lower PM while performing elbow extension motion is given. The length of legs is in full extended position during elbow extension motion, compared to flexion motion. In inverse displacement analysis of 2SPR-type lower PM, when the 2SPR-type lower PM performs elbow flexion to extension motions, the displacement of the top platform $m$ varies smoothly, the length of legs $r_{i 1}$ $(i=1,2,3)$ are varied smoothly. Thus, the 2SPR-type lower PM has good kinematic characteristics (displacement).

Table 9. Length of legs of lower PM during inverse displacement analysis of elbow extension motion

\begin{tabular}{|c|c|}
\hline $\begin{array}{c}\text { Length of leg } r_{11} \\
{[\mathrm{~mm}]}\end{array}$ & $\begin{array}{c}\text { Length of leg } r_{21} \\
{[\mathrm{~mm}]}\end{array}$ \\
\hline 255.62 & 255.62 \\
\hline
\end{tabular}

In Table 10, the length of the legs of lower PM during elbow pronation motion is given.

Table 10. Length of legs of lower PM during inverse displacement analysis of elbow pronation motion

\begin{tabular}{|c|c|}
\hline $\begin{array}{c}\text { Length of leg } r_{11} \\
{[\mathrm{~mm}]}\end{array}$ & $\begin{array}{c}\text { Length of leg } r_{21} \\
{[\mathrm{~mm}]}\end{array}$ \\
\hline 187.135 & 187.135 \\
\hline
\end{tabular}

In Table 11, the length of the legs of lower PM during elbow supination motion is given. During elbow pronation and supination motions, the manipulator legs maintain same length. There is no translational movement of the top platform in $z$-axis. The gear drive in lower PM manipulator will perform elbow pronation and supination motion.
Table 11. Length of legs of lower PM during inverse displacement analysis of elbow supination motion

\begin{tabular}{|c|c|}
\hline Leg $r_{11}$ length [mm] & Leg $r_{21}$ length [mm] \\
\hline 187.135 & 187.135 \\
\hline
\end{tabular}

In Tables $8-11$ express the length of legs during displacement of lower parallel manipulator. During elbow pronation and supination motions, the length throughout the motion remains constant. During elbow flexion and extension motion, the degree of variation in rotation angle is more, hence, the rotation angle during the extreme positions of elbow flexion and extension motions are only taken to calculate the length. Therefore, one value in each table mean the final value during movement.

In the case of elbow pronation and supination motions, the lower PM has good kinematic characteristics (displacement). It means that there is no singular configuration for the lower parallel manipulator during the desired motion.

In Figure 8, during inverse velocity analysis of elbow pronation to supination motion, when the velocity of the top platform varies smoothly, the velocities of the legs varies smoothly. Thus, the 2SPR-type lower PM has good kinematic characteristics (velocity).

Based on Figure 9, it can be understood that the lower PM also has good kinematic characteristic (velocity) during elbow flexion to extension motion.

During forward velocity analysis, it was not possible to find out the velocity of the top platform from known velocities of legs. On computation, Matlab provided the comment "The jacobian matrix was singular to working precision". It means that the determinant of the jacobian matrix is zero and inverse of the jacobian matrix is infinity. Thus, manipulator singularity happens while performing rehabilitation. Hence, inverse acceleration analysis is not possible in any of the elbow motions. Thus, it can be concluded that the 2SPR-type lower PM cannot be used for rehabilitation of elbow motions. Hence, another manipulator needs to be designed for performing elbow pronation, supination, flexion and extension motions.

Finally, it can be concluded from Figs. 5-9 that during inverse velocity analysis, when the velocity or acceleration near the center of the top platform vary smoothly, the velocities or accelerations of the active legs vary smoothly. Similarly, during forward kinematic analysis, when the velocities or accelerations of the legs vary smoothly, the velocity or acceleration 


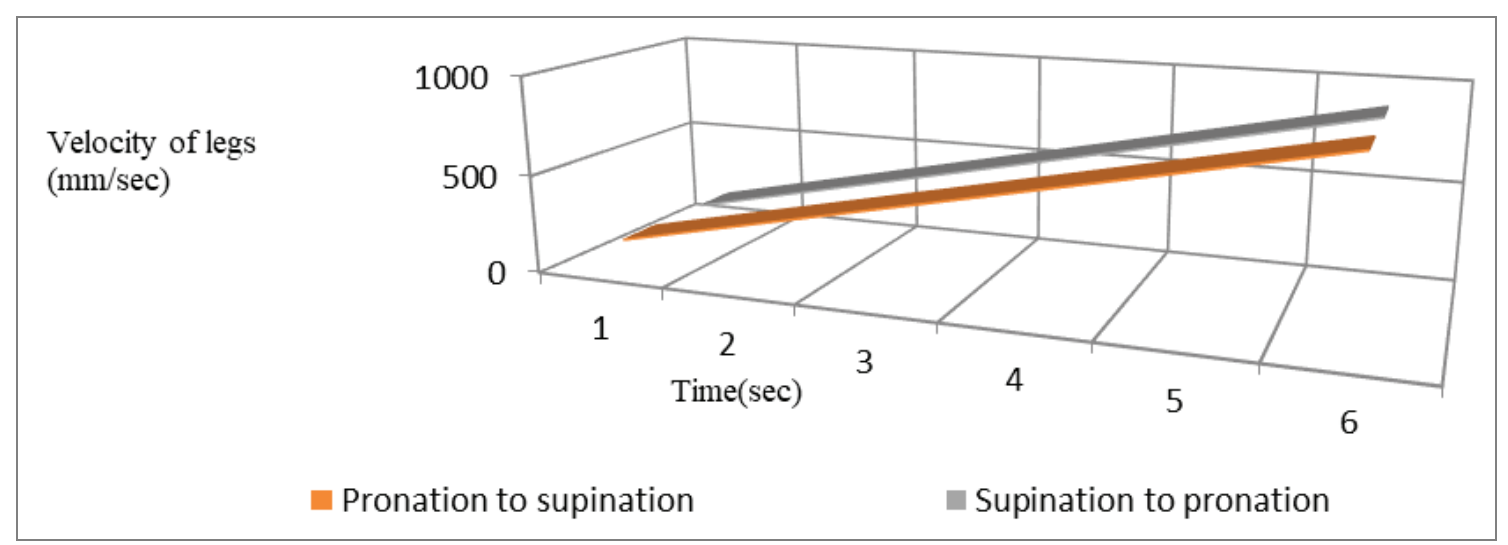

Fig. 8. Inverse velocity (legs) versus time for lower PM (pronation and supination) during elbow motions

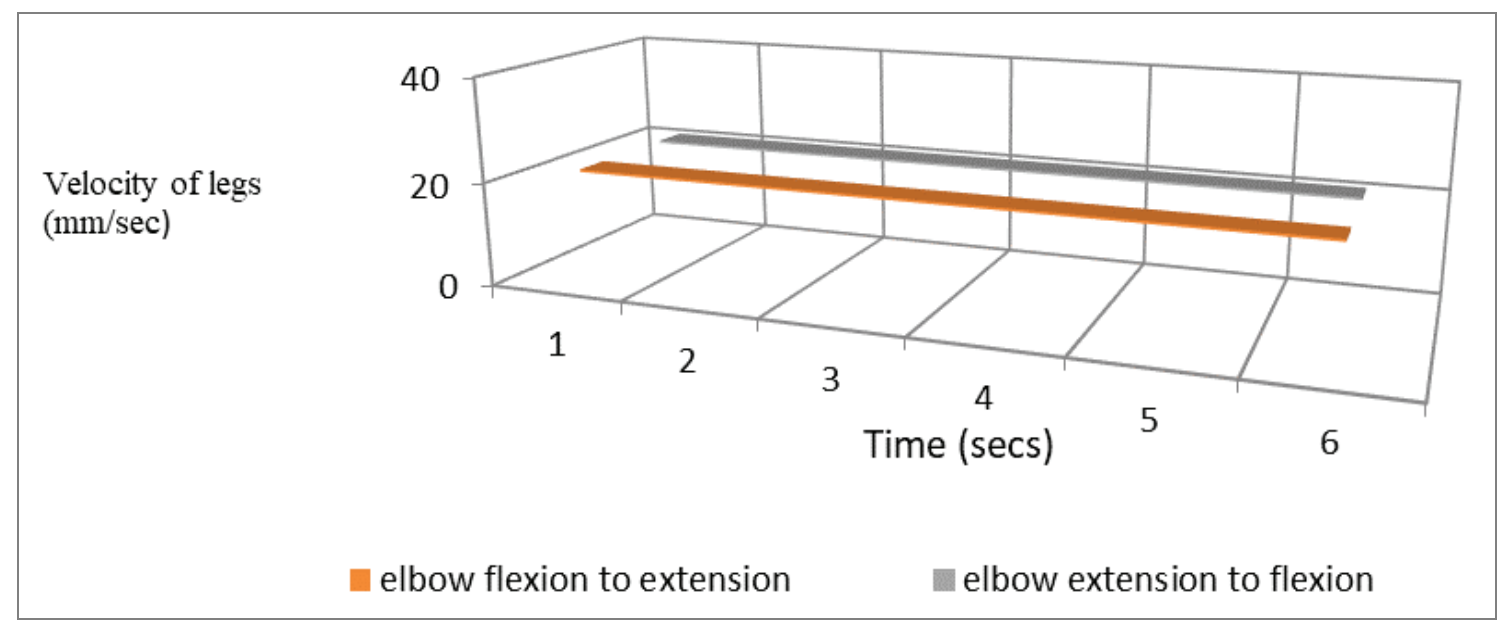

Fig. 9. Inverse velocity (legs) versus time for lower PM (flexion and extension) during elbow motions

near the center of the top platform vary smoothly. Thus, during forward and inverse kinematics analysis, good kinematic characteristics (displacement, velocity and acceleration) are observed for upper PM and not for lower PM.

The positioning workspace of the 3SPS-type and 2SPR-type parallel manipulators in $X Y$-plane are plotted and shown in Fig. 10. The procedure adopted in Section 2.6 to find the workspace produces two circles for each of the active legs. For example in Fig. 10a during flexion/extension motions of the wrist, circles $C_{1 X}$, and $C_{1 N}$ are produced by the first leg. Similarly, $C_{2 X}, C_{2 N}$ are produced by the second leg and $C_{3 X}$ and $C_{3 N}$ are produced by the third leg. Intersection of all 6 circles at the same time is the positioning workspace of the center point $\left(o_{2}\right)$ of the top platform of upper PM. In Table 4, since $r_{1}$ and $r_{2}$ are same, two circles $\left(C_{1 X}, C_{2 X}\right.$ and $\left.C_{1 N}, C_{2 N}\right)$ are identical in Fig. 10a.

During wrist radial deviation motion and ulnar deviation motion, as it is known from inverse dis- placement analysis, the length of the third leg of the 3SPS-type parallel manipulator remain constant, hence, $C_{3 X}$ and $C_{3 N}$ circles are identical. Thus, overall 5 circles can only be seen in Fig. 10b. In Figs. 10a, b, since all circles are not identical, they will have a common intersection point, which is the positioning workspace in case of all wrist motions. The 2SPRtype PM has two legs, thus, there are 4 circles $\left(C_{11}\right.$, $C_{12}-\operatorname{leg} 1$ and $C_{21}, C_{22}-\operatorname{leg} 2$ ) for elbow motions. During elbow flexion and extension motions, both legs change length in the same way. Therefore only two circles out of total 4 circles are visible in Fig. 10c. In Fig. 10d, all 4 circles are identical during elbow pronation and supination motions since length of both legs does not vary as gear drive performs the desired motion. Thus, the positioning workspace of the center point $(o)$ of the top platform of lower PM in $X Y$-plane are obtained. In Fig. 10c, the whole area within $C_{12}$ and $C_{22}$ is the workspace and in Fig. 10d, the center point of all four circles is the positioning workspace. 


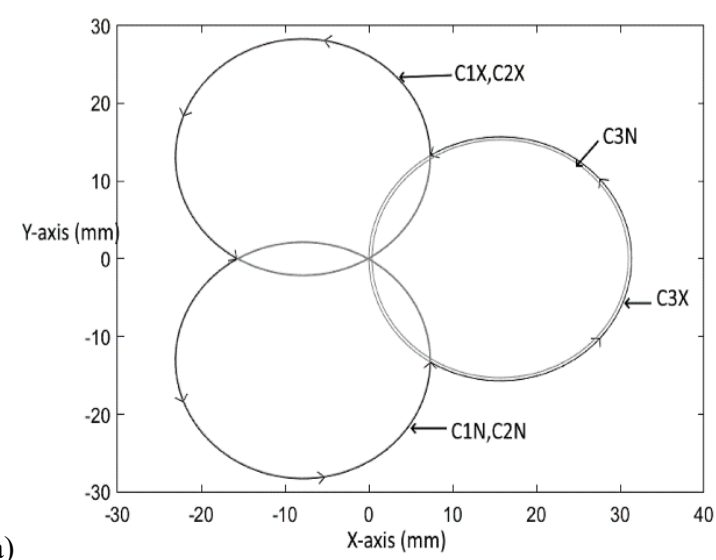

(a)

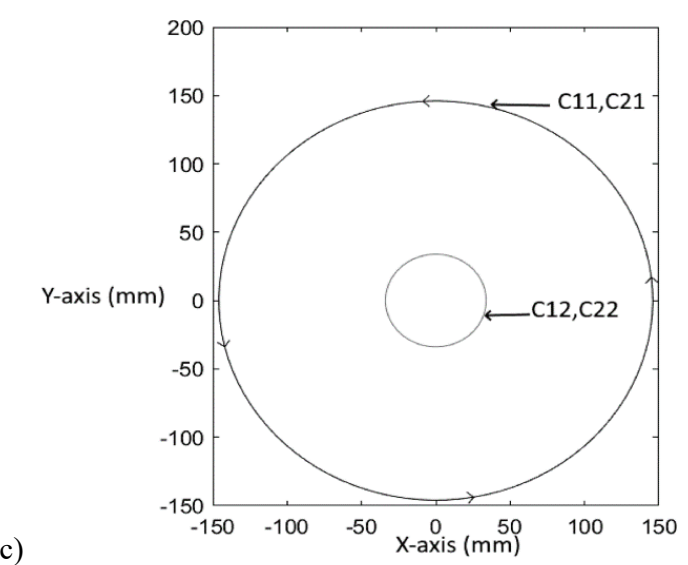

(b)

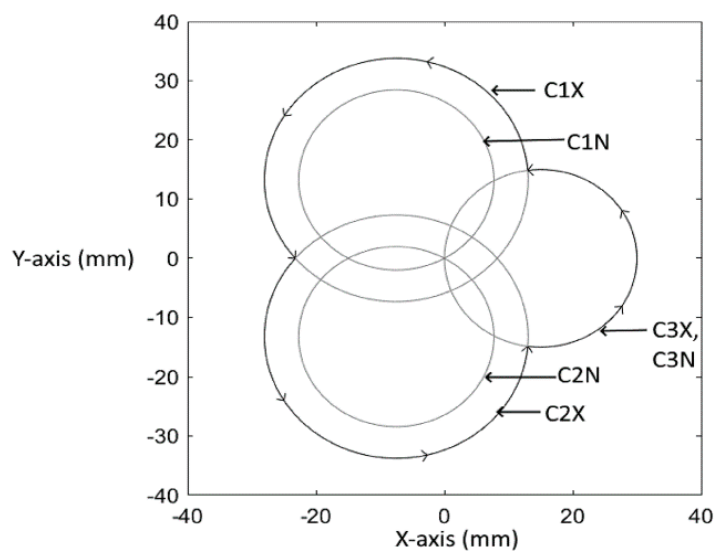

(d)

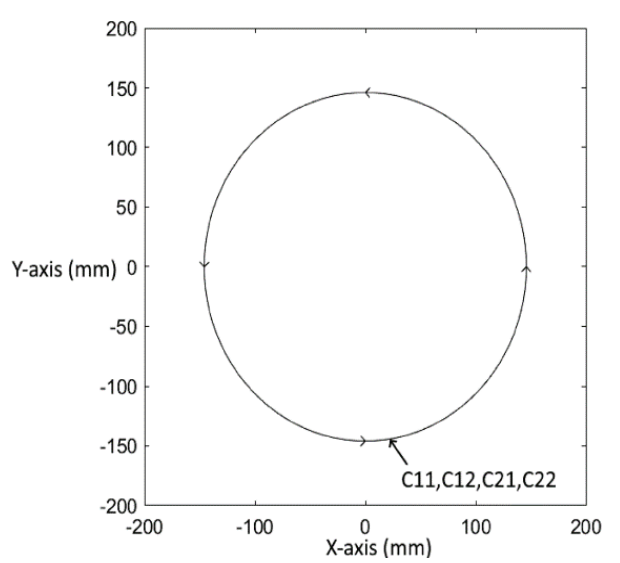

Fig. 10. Workspace analysis: (a) wrist flexion and extension, (b) wrist radial deviation and ulnar deviation, (c) elbow flexion and extension, (d) elbow pronation and supination

\section{Discussion}

The objective of this study was to design a wearable robotic device during upper limb rehabilitation for patients affected by stroke. The proposed rehabilitation device is a novel device in terms of its kinematic structure. This robot has a hybrid kinematic structure since it has two parallel manipulators connected in series. In this project, an attempt was made to solve its kinematics during its performance. The upper parallel manipulator structure has 3SPS legs and lower parallel manipulator structure has 2SPR legs. This device is connected to human upper limb and rehabilitation was performed by simulation. The range of motion during wrist flexion, wrist extension, wrist ulnar deviation, wrist radial deviation, elbow flexion, elbow extension, elbow pronation, and elbow supination motions was found out. Scientist normally use sensors for kinematic measurements, just like the kinematic variables of the lower limb was measured using retro reflective markers [5]. In this project, the point of contact between the exoskeleton robot and upper limb was noted for obtaining the range of motion via simulation.

In Table 2, the capabilities of the human elbow and wrist joints and for the corresponding exoskeleton joints are shown. The requirements of the workspace closely resemble the normal ROM of human joints. The exoskeleton design meets the specified workspace specifications for elbow joint with the exception of the wrist joint. It should be noted that the elbow workspace available is adequate for many routine jobs. In elbow flexion/extension, $70 \%$ of ROM can be recreated, $100 \%$ during elbow pronation and $83 \%$ during elbow supination. Nearly $20 \%$ of wrist flexion ROM and $54 \%$ of wrist radial deviation ROM may be replicated. It is only $15 \%$ for wrist ulnar deviation and $7 \%$ for wrist extension. Employing a 3SPS-type PM to perform wrist motions, when compared to the physiological ROM for the corresponding anatomical joint, is far too low. The key benefit proved in 3SPS-type PM is that it has good kinematic characteristics and mechanical singularity did not develop within the robot wrist workspace. The drawback in 2SPR-type PM is that it has poor kinematic characteristics and a singu- 
larity in workspace which must be avoided via good trajectory planning.

It is difficult to match the mechanical joint ROM of an exoskeleton robot to that of a human arm. The majority of upper limb exoskeleton robot mechanical designs have been evaluated by analysing the robot's functionalities. The mechanical singularity should not exist within the robots workspace, and this should be taken into account when designing. As a result, the exoskeleton device must allow for natural human arm movements while reducing the workspace of the human arm to a minimum. Researchers found that the post-stroke patients have lower gait cadence compared to a healthy person [7]. Thus, the proposed rehabilitation device can improve the quality of life of the stroke patients by regaining the muscle strength through rehabilitation therapy using robots. Scientists and researchers have found that modern technologies can produce high impact on rehabilitation treatment and are effective for stroke-affected patients as well [6].

\section{Conclusion}

3D model of the proposed hybrid (serial-parallel) robot manipulator was constructed and simulated using Solidworks software package. It is composed of an upper 3SPS-type and a lower 2SPR-type parallel manipulators. It performs two wrist motions and two elbow motions which a human arm performs for doing Activities of Daily Living (ADL). The formula for solving the kinematics of the hybrid robot were derived. The positioning workspace of the serial-parallel robotic arm was computed using Matlab.

If the displacement, velocity and acceleration of the top platform of upper and lower parallel manipulators are given, it is possible to solve the displacement, velocity and acceleration of the active legs. The 3SPS-type parallel manipulator has good kinematic characteristics (displacement, velocity and acceleration) while performing wrist motions. Thus, 3SPS-type parallel manipulator is suitable for usage in performing rehabilitation therapy in stroke-affected patients. The 2SPR-type parallel manipulator produced singular configuration while performing the desired rehabilitation elbow motions. Thus, 2SPR-type parallel manipulator is not suitable for usage in performing rehabilitation therapy in stroke-affected patients.

The drawback in 2SPR-type parallel manipulator is that it has a singularity in workspace. In certain manipulators with proper trajectory planning the singularity can be avoided and hence this needs to be investi- gated in the proposed 2-SPR manipulator in future. If good kinematic characteristics cannot be achieved by trajectory planning, the 2SPR-type parallel manipulator should be replaced with another parallel manipulator by including more spherical joints. In mechanical systems such as robotic wrists, orientation mechanisms, exoskeletons, and so on, spherical motion is common. Those systems are designed to provide three degrees of absolute rotational freedom. Also, a mechanism which could perform 3-degree-offreedom for shoulder motions is to be designed and attached to the existing hybrid robot, so that the robot will perform all degrees-of-freedom which a human upper limb has. The future scope of study will be taking all these into consideration.

\section{Acknowledgements}

The authors would like to thank Coimbatore Institute of Technology, Coimbatore for providing the facilities to carry out the project.

\section{References}

[1] Byl N.N., Abrams G.M., Pitsch E., Fedulow I., Kim H., SIMKINS M., Chronic stroke survivors achieve comparable outcomes following virtual task specific repetitive training guided by a wearable robotic orthosis (UL-EXO7) and actual task-specific repetitive training guided by a physical therapist, Hand Ther., 2013, 26, 343-352.

[2] Gosselin C., Determination of the Workspace of 6-DOF Parallel Manipulators, J. Mech. Des., 1990, 112 (3), 331-336.

[3] Gupta A., O’Malley M.K., Design of a Haptic Arm Exoskeleton for Training and Rehabilitation, IEEE ASME Trans. Mechatron., 2006, 11 (3), 280-289.

[4] Gupta A., O’Malley M.K., Patoglu V., Design, Control and Performance of Rice Wrist: A Force Feedback Wrist Exoskeleton for Rehabilitation and Training, Int. J. Rob. Re., 2008, 27 (2), 233-251.

[5] JiAng X., Chen H., Sun D., BAKer J.S., Gu Y., Running speed does not influence the asymmetry of kinematic variables of the lower limb joints in novice runners, Acta Bioeng. Biomech., 2021, 23 (1), 69-81.

[6] KabaŁa T., Sawko L., Dziuba-SŁonina A., Giemza C., Influence of modern technologies used in rehabilitation on selected functional parameters of the spine of patients with low back pain, Acta Bioeng. Biomech., 2020, 22 (4), 101-107.

[7] Kowal M., Kolcz A., Dymarek R., Paprocka-Borowicz M., GNUS J., Muscle torque production and kinematic properties in post-stroke patients: a pilot cross-sectional study, Acta Bioeng. Biomech., 2020, 22 (1), 11-20.

[8] Loureiro R.C.V., Harwin W.S., LAmperd R., Collin C., Evaluation of reach and grasp robot-assisted therapy suggests similar functional recovery patterns on proximal and distal arm segments in sub-acute Hemiplegia, IEEE Trans. Neural. Syst. Rehabilitation Eng., 2014, 22 (3), 593-602. 
[9] Lu Y., DAi Z., Ye N., WANG P., Kinematics/statics analysis of a novel serial-parallel robotic arm with hand, J. Mech. Sci. Technol., 2015, 29 (10), 4407-4416.

[10] LUKANIN V., Inverse kinematics, forward kinematics and working space determination of 3-dof parallel manipulator with S-P-R joint structure, Periodica Polytechnica Ser. Mech. Eng., 2005, 49 (1), 39-61.

[11] Martinez J.A., NG P., Lu S., Campagna M.S., Celik O., Design of Wrist Gimbal. A forearm and wrist exoskeleton for stroke rehabilitation, 2013 IEEE International Conference on Rehabilitation Robotics, Seattle, Washington USA, (June 24-26, 2013).

[12] Milot M.H., Spencer S.J., Chan V., Allington J.P., Klein J., Chou C., Bobrow J.E., Cramer S.C, ReInKensmeyer D.J., A crossover pilot study evaluating the functional outcomes of two different types of robotic movement training chronic stroke survivors using the arm exoskeleton bones, J. Neuroeng. Rehabil., 2013, 10 (112), 1-12.

[13] Nef T., Minelu M., Colombo G., Riener R., ARMin - Robot for rehabilitation of the upper extremities, Proceedings of the IEEE International Conference on Robotics and Automation, Orlando, FL, USA, (May 15-19, 2006), 3152-3157.

[14] OtaKa E., OtaK Y., Kasuga S., Nishimoto A., YamazaKi K., KAWAKAMI M., UshiBA J., LIU M., Clinical usefulness and validity of robotic measures of reaching movements in hemiparetic stroke patients, J. Neuroeng. Rehabil., 2015, 12, 1-10.

[15] Palermo E., Hayes D.R., Russo E.F., Calabrò R.S., PACILLI A., FILONI S., Translational effects of robot-mediated therapy in subacute stroke patients: an experimental evaluation of upper limb motor recovery, Peer J., 2018, 6, 1-25.
[16] Perry J.C., Rosen J., Burns S., Upper-limb powered exoskeleton design, IEEE/ASME Trans. Mechatron., 2007, 12, 408-417.

[17] Pineda-Rico Z., Sanchez De Lucio J.A., Lopez F.J.M., CRUZ P., Design of an exoskeleton for upper limb robot - assisted rehabilitation based on co-simulation, J. Vibroengineering, 2016, 18 (5), 3269-3278.

[18] Pons J.L., Wearable robots: Biomechatronic Exoskeletons, John Wiley \& Sons, Ltd., 2008.

[19] Rahman M.H., Rahman M.J., Cristobal O.L., SaAD M., Kenné J.P., ARchambault P.S., Development of a whole arm wearable robotic exoskeleton for rehabilitation and to assist upper limb movements, Robotica, 2015, 33 (1), 19-39.

[20] Tsai L.W., Robot Analysis: The Mechanics of Serial and Parallel Manipulator, John Wiley \& Sons, 1999.

[21] Vaida C., Plitea N., Carbone G., Birlescu I., Ulinici I., Pisla A., Pisla D., Innovative development of a spherical parallel robot for upper limb rehabilitation, Int. J. Mech. Robot. Syst., 2018, 4 (4), 256-276,

[22] TAPPeiner L., OtTAViano E., Husty M.L., A Cable-Driven Robot for Upper Limb Rehabilitation Inspired by the Mirror Therapy, Springer-Mechanisms and Machine Science Book Series, 2017, 174-181.

[23] Alamdari A., Krovi V., Parallel articulated-cable exercise robot (pacer): novel home-based cable-driven parallel platform robot for upper limb neuro-rehabilitation, Proceedings of the ASME International Design Engineering Technical Conferences and Computers and Information in Engineering Conference, Boston, Massachusetts, USA, (August 2-5, 2015), 1-10. 